\title{
Development and Validation of a Social Anxiety Scale (SAS)
}

\author{
Erka T. Hutama ${ }^{\text {ab }}$, James Atyeo ${ }^{\mathrm{b}}$, \& Ike Anggraika Kuntoro ${ }^{\mathrm{c} *}$
}

${ }^{a}$ Faculty of Psychology, Universitas Indonesia, Depok, Indonesia; ${ }^{b}$ School of Psychology, University of Queensland, Brisbane, Queensland, Australia; ${ }^{c}$ Department of Developmental Psychology, Faculty of Psychology, Universitas Indonesia, Depok, Indonesia

*Corresponding Author:

Ike Anggraika Kuntoro

Developmental Psychology Department

Faculty of Psychology, Universitas Indonesia

Jl. Lkr. Kampus Raya, Depok, Jawa Barat

Indonesia, 16424

Tel.: +62 217270004

Email address: ike.anggraika@ui.ac.id 


\title{
Development and validation of Social Anxiety Scale (SAS)
}

\begin{abstract}
Although several scales have been developed to measure social anxiety, none of these scales have focused on measuring social anxiety in the college setting. This study aimed to develop a scale to measure social anxiety that can be used specifically for college students. The scale was developed based on previously published scales of social anxiety (social phobia disorder). A total of 127 psychology undergraduate students (92 females and 35 males), aged between 18 and 51 years $(M=22.05 ; S D=3.91)$, participated in this work. To validate our scale, we conducted concurrent validity analysis by correlating the proposed social anxiety scale (SAS) with established scales. The participants answered questions related to five measures, including a demographic and pro-social questionnaire, SAS, Extraversion Scale, Intolerance of Uncertainty Scale, and Student Adaptation to College Questionnaire. We hypothesized that social anxiety would be (1) negatively correlated with extraversion, (2) positively correlated with intolerance of uncertainty, (3) negatively correlated with student adaptation to college, and (4) positively correlated with pro-social behavior (PB). This study found that all of the hypotheses could be confirmed, except that for PB. Our SAS was further found to possess excellent internal consistency (Cronbach's $\alpha=.86$ ), and discriminant analysis of the related items yielded satisfactory results. Our findings suggest that the new scale is a reliable and valid tool for measuring social anxiety among college students.
\end{abstract}

Keywords: Scale development; Social anxiety; Social anxiety scale

\section{Introduction}

As stated in the Diagnostic and Statistical Manual of Mental Disorder V (APA, 2013), social anxiety or social phobia disorder is defined as a fear of social situation(s) and a fear of doing something humiliating or embarrassing in a social engagement as shown by anxiety symptoms (American Psychiatric Association, 2013). An individual may be considered to have social anxiety if the problems are consistent for at least 6 months (Antony \& Swinson, 2000). Social anxiety disorder is classified into several sub-types in the DSM-V, including: (1) circumscribed (fear of only one or two social situations), (2) generalized (fear of most social situations), and (3) non-generalized (clinically significant anxiety in social situations with at least one broad domain of social functioning that is not associated with significant anxiety) (Heinberg \& Holt, 1989; as cited by Anthony \& Swinson, 2000). Other subtypes proposed by the Task Force in DSM-IV consist of (a) performance (e.g., public speaking, eating in public), (b) limited interactional (e.g., dating, speaking to strangers), and (c) generalized (phobic stimuli in most social situations; as cited by Antony \& Swinson, 2000). Mattick and Clarke (1998) posited that social anxiety refers to distress when meeting or talking to someone; this anxiety is rooted in fear of being inarticulate, sounding stupid, being boring, not knowing what to say or how to respond to social interaction, and being ignored.

Social anxiety is considered one of the main factors influencing an individual's social interaction behaviors, including pro-social behavior (PB; e.g., helping a friend, giving a spare change to those in need) (Culotta \& Goldstein, 2008). Social anxiety has been found to be negatively correlated with extraversion and agreeableness (Kaplan, Levinson, Rodebaugh, Menatti, \& Weeks, 2015), which is considered to be the interplay of traits and motives when helping others 
(Carlo, Okun, Knight \& Guzman, 2004). Carlo et al. (2004) argued that extraversion and agreeableness predict PB (see Graziano \& Eisenberg, 1997). In particular, social anxiety has been predicted to be negatively correlated with PB. These previous studies have motivated us to develop a social anxiety scale (SAS) that can explain the relationship between anxiety and prosocial engagement with good reliability and validity.

Several scales have been used to measure social anxiety as a trait. Two measures of social anxiety are the Social Phobia and Anxiety Inventory (SPAI; Turner, Beidel \& Dancu, 1996) and Social Phobia Scale (SPS; Mattick \& Clarke, 1998). Despite their benefits, however, the SPAI and SPS did not perform well in discriminating between social anxiety and other anxiety disorders, thereby demonstrating that they have poor validity (Peters, 2000). To clarify results, Peters (2000) explained that the SPAI and SPS failed to discriminate social phobia and panic disorder with or without agoraphobia (i.e., an irrational or excessive fear of open or public places), which means social anxiety has yet to be clearly defined, especially as a mental state. Both the SPAI and SPS provided inaccurate representations of anxiety measurement as they cannot successfully differentiate general anxiety and specific phobias that may occur in social environments/cues. These findings illustrate why further modification of the current measures is critical in determining the condition of a patient to provide proper interventions or effective treatments.

While most of the research on the topic involves clinical samples (e.g., Peters, 2000) or is only partially concerned with non-clinical groups (e.g., Santos, Loureiro, Crippa \& Osório, 2015), we believe in the importance of more general, non-clinical groups in the population. In the academic setting, up to $20 \%$ of all college students are reported to experience psychological problems including anxiety (Kumaraswamy, 2013), and the social environment has been proposed to affect students' anxiety toward adjusting in the academic setting (Crede \& Niehorster, 2012). As per the current literature, we believe in the importance of determining the social anxiety sub-traits that may emerge in the academic setting. Therefore, we intend to modify the SPAI and SPS to construct a newer, more-comprehensive, but simpler SAS for non-clinical samples, specifically college students, in the academic setting. We further aim to examine the reliability and validity of the proposed scale and expect to achieve a measure high internal consistency.

Previous studies have determined that social anxiety is negatively correlated with intolerance of uncertainty and highly correlated with fear and anxiety (Carleton, Norton \& Asmundson, 2007). Hence, we hypothesized that social anxiety is correlated with intolerance of uncertainty. In a meta-analytic review, student adjustment in college was found to be correlated with the social environment interaction of students (Crede \& Niehorster, 2012). Thus, as social environment interaction is considered a sub-trait in predicting social anxiety, we hypothesized that social anxiety will be negatively correlated with student adjustment.

\section{Methods}

\section{Participant}

This study involved 127 participants (92 females and 35 males) who were university students enrolled in a PSYC3020 (Measurement in Psychology) class at the University of Queensland. The age of the participants ranged from 18 years to 51 years $(M=22.05, S D=3.91)$. The participants were invited during tutorial class to fill an in-class online questionnaire and scales. 
Prior to data collection, demographic questions, including those pertaining to age and gender, were obtained to gain general information on the participants. Subjects participated voluntarily, and the questions of our research were published in the university's learning website, possibly affecting the numbers of participants who were willing to fill the questionnaires and eventually decided to withdraw from the study.

\section{Design}

Correlation analysis was conducted to examine the concurrent validity of SAS with four other validating scales.

\section{Measures}

Five research instruments were applied in the present study:

Pro-social Behavior. We posted a question to obtain insights into the general PB of the participants. A pilot study was conducted among the researchers to establish whether the items could represent pro-social acts in general. PB was determined along with the demographic questions. Participants were asked whether they had "engaged in any act today that involved helping someone else or doing something good for a good cause." This measure was scaled between 1 (yes) or 2 (no).

Social Anxiety Scale. We constructed a SAS based on a previously developed scale of the SPAI (Turner, Beidel \& Dancu, 1996) and SPS constructed by Mattick and Clarke (1998) using 10 items. The scale consisted of items that aimed to indicate an individual's social anxiety level. After each item was constructed, we conducted a pilot study to ensure that the items represented the constructs of social anxiety. We removed and edited unsuitable items based on the results of the pilot study. The final version of the scale consisted of ten items (see Table 1), and all items were ranked via a 5-point Likert-scale. Two out of 10 final items were reverse-scored (e.g., "I find it easy to talk to people I've just met"). The participants were asked to rate statements from 1 $=$ strongly disagree to $5=$ strongly agree. We found that this scale had high reliability (Cronbach's $\alpha=.85$ ).

Table 1.

Final Items of the Social Anxiety Scale

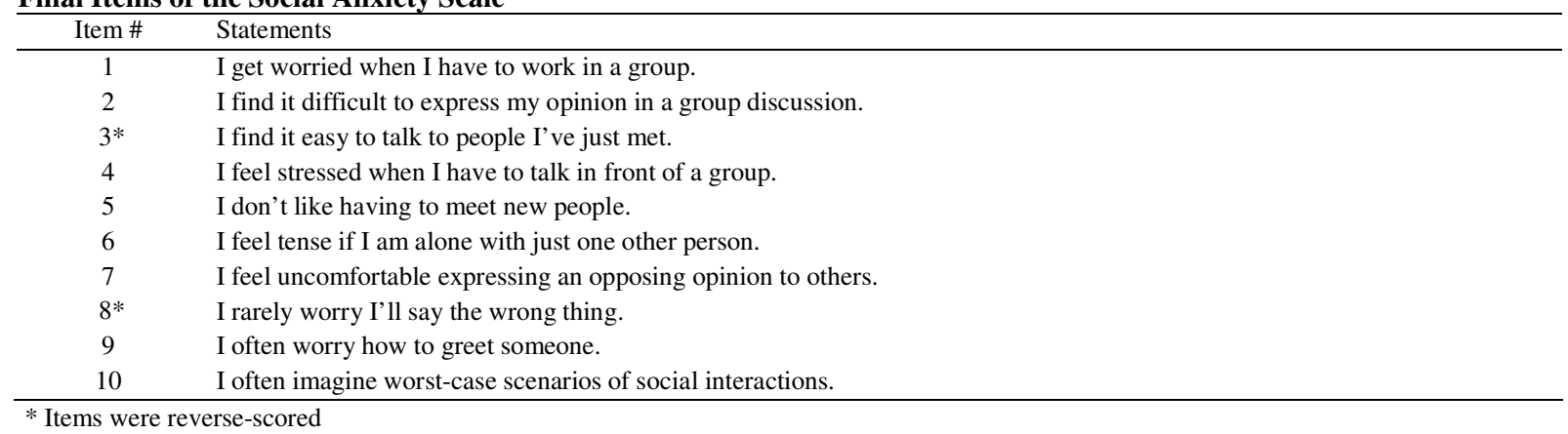

Extraversion Scale (ES). Based on the Two-Dimensional Measurement (TDM) scale developed by Eysenck (1958), we measured participant extraversion with six questions. The items posted in the scale included "Would you rate yourself as a lively individual?," "Are you a talkative person?," "Do you take initiative in making new friends?," and "Are you quiet when you are with 
other people?" For this study, we selected specific measurements for individual extroversion and removed neuroticism-related items in the original TDM. Participants were asked to choose between $1=$ yes or $2=n o$, depending on whether the statements represented them. The reliability of this scale was found to be acceptable (Cronbach's $\alpha=.61$ ).

Intolerance of Uncertainty Scale. For this validating scale, a short version of the Intolerance of Uncertainty Scale (IUS-12; suggested by Carleton et al., 2007) was used. This scale measured individual tolerance against unpredicted or unplanned situations. Twelve items were posted in the scale, including "Unforeseen events upset me greatly," "It frustrates me not having all the information I need," One should always look ahead so as to avoid surprises," "Uncertainty keeps me from living a full life," and "When I am uncertain I can't function very well." Participants were asked to rate how the statement in the item represented their characteristics using a 5-point Likert-style ranging from $1=$ not at all characteristic of me to $5=$ entirely characteristic of me. This scale had very good reliability (Cronbach's $\alpha=.86$ )'

Student Adjustment to College Questionnaire. The Student Adjustment to College Questionnaire (SACQ) developed by Baker \& Siryk (1989) was adapted and used in this work to validate social anxiety. The original questionnaire consisted of 66 items, divided into two sub-scales, i.e., Academic Adjustment and Social Adjustment. In the present study, 12 out of 24 items in the subscale Academic Adjustment were posted. The items selected as the validating scale included " $M y$ academic goals and purposes are well defined," "I enjoy academic works," and "I attend classes regularly." The items were chosen according to their relevance to the SAS and aimed to avoid repetition and redundancies, which may influence the participants' responses. This measure used a 9-point Likert-style ranging from $1=$ does not apply to me at all to $9=$ applies very closely to me. This questionnaire was found to be reliable (Cronbach's $\alpha=.76$ ).

\section{Procedure}

The participants were first asked to complete a series of tests in the tutorial class they attended and then asked to fill a consent form prior to answering the questionnaires; they were further informed that they could withdraw from the research at any time they chose. The participants completed the questionnaires individually using the computers provided in their classes, and data were collected within one week of the participants' tutorial classes. Online questionnaires were also provided in the UQ Blackboard website for participants who were unable to attend classes during data collection week. Participants who filled questionnaires online were also provided a briefing and consent sheet.

\section{Data Analysis}

Descriptive statistical analysis was conducted to characterize the demographic data. We then conducted the parametric Spearman $r$ correlation test on the SAS and all validating scales using SPSS. To assess the reliability of the scale, we measured its Cronbach's $\alpha$. Item discrimination analysis was also conducted.

\section{Results}

Statistics for sample means, standard deviations, age ranges, PB, SAS, and all three validating scales (ES, IUS-12, and SACQ) are presented in Table 2. 
Table 2

Descriptive Statistics for Age, Gender, and Pro-Social Behavior (PB)

\begin{tabular}{|c|c|c|c|c|c|}
\hline & $M$ & $S D$ & Minimum & Maximum & Skew \\
\hline Age & 22.05 & 3.30 & 18 & 51 & 4.44 \\
\hline Gender & 2.51 & 0.76 & 1.00 & 4.30 & 1.02 \\
\hline PB & 1.53 & 0.50 & 1 & 2 & -0.11 \\
\hline SAS & 26.20 & 6.68 & 10.10 & 41.40 & -0.14 \\
\hline ES & 0.01 & 3.31 & -5.17 & 5.17 & 0.04 \\
\hline IUS-12 & 33.62 & 7.44 & 14.08 & 49.33 & -0.16 \\
\hline SACQ & 64.44 & 12.94 & 25.08 & 95.75 & -0.30 \\
\hline
\end{tabular}

Note. ES = Extroversion Scale; PB = Pro-social Behavior; IUS-12 = Intolerance of Uncertainty Scale; SACQ = Student Adjustment to College Questionnaire

Our analysis revealed a significant negative correlation between SAS and ES, thereby indicating that higher social anxiety correlates with lower extraversion, $r(124)=-.39, p<.001$. As we predicted in our first hypothesis, a higher SAS score indicates a lower ES score. Our second hypothesis was also supported, which means SAS scores are positively correlated with higher IUS, significant at $r(120)=.56, p<.001$. SAS was also found to be negatively correlated with SACQ, thereby proving our third hypothesis, at $r(124)=-.37, p<.001$. For our final hypothesis, we conducted Spearman $r$ correlation analysis to determine whether social anxiety is a predictor of $\mathrm{PB}$. However, the result we obtained was not significant, $r(12)=.06, p=.503$, which means PB does not indicate low or high social anxiety. Table 3 lists the intercorrelations between SAS and the other validating scales.

Table 3

Summary of Intercorrelations between PB and SAS, ES, IUS-12, andSACQ

\begin{tabular}{lccccc}
\hline & 1 & 2 & 3 & 4 & 5 \\
\hline 1. PB & - & .05 & -.14 & .06 & $-.18^{*}$ \\
2. SAS & .06 & - & $-.39 * *$ & $.5^{* *}$ & .10 \\
3. ES & -.14 & $-.39 * *$ & - & $-.37^{* *}$ & .04 \\
4. IUS-12 & .06 & $\mathbf{5 6 * *}$ &.$- .37 * *$ & - & .00 \\
5. SACQ & -.15 & .10 & .04 & .00 & - \\
\hline
\end{tabular}

*Correlation is significant at the 0.05 level (2-tailed).

**Correlation is significant at the 0.01 level (2-tailed).

Item discrimination indices were calculated to determine the extent of an item's ability to differentiate low and high scorers among the participants. The scores were divided into two groups to differentiate how they scored in the SAS: the low percentile group $(33 \% ; \mathrm{n} L)$ and the high percentile group $(33 \%$; $\mathrm{n} U)$. Afterward, we summarized the total participants in the highscoring group $(U)$ to count the number of participants who answered the items correctly; we repeated these same procedures in in the low-scoring group $(L)$. The item discrimination index scores $(d)$ were obtained by subtracting $U$ divided by $\mathrm{n} U$ with $L$ divided by $\mathrm{n} L$ (Ary, Jacobs, Irvine \& Walker, 2013). This formula was repeated for each item and cross-tabulated to examine the discrimination index score of each item. A high $d$ score indicates that all high scorers understood the item correctly, and a low $d$ score indicates a misperception of the items presented. 
Table 4

Item Discrimination Indices for the SAS

\begin{tabular}{llll} 
& $\boldsymbol{U}$ & $\boldsymbol{L}$ & $\boldsymbol{D}$ \\
\hline Item 1 & 7 & 44 & 0.63 \\
Item 2 & 2 & 41 & 0.62 \\
Item 3* & 0 & 38 & 0.57 \\
Item 4 & 10 & 25 & 0.65 \\
Item 5 & 2 & 24 & 0.50 \\
Item 6 & 4 & 45 & 0.36 \\
Item 7 & 1 & 12 & 0.43 \\
Item 8* & 11 & 45 & 0.61 \\
Item 9 & 16 & 44 & 0.65 \\
Item 10 & 2 & 31 & 0.46 \\
\hline
\end{tabular}

Note. $\mathrm{n} L=44$ and $\mathrm{n} U=42$ for all items. * Items were reverse-scored

Table 4 demonstrates that, with the exception of item \#6, all of the items in the scale scored above 0.40 , which means they can be considered good measures. Based on these indices, we can safely assume that the items in the scale can represent sub-traits of the social anxiety construct. Considering an average mean $d$ score of 0.48 , this scale should be able to correctly differentiate levels within the SAS.

\section{Discussion}

The aim of this study was to construct a new brief scale that can accurately measure social anxiety in PB. We determined four hypotheses related to our newly constructed scale. First, we hypothesized that social anxiety is negatively correlated with extraversion. Our second and third hypotheses are that social anxiety are positively correlated with intolerance of uncertainty and negatively correlated with college student adjustment, respectively. Finally, we hypothesized that social anxiety is able to predict PB, which means it is able to correctly measure an individual's social anxiety level. As explained in the previous literature, social anxiety is negatively correlated with extraversion. The results of our study confirmed that an individual scoring high in social anxiety will also score low in extraversion. Moreover, we successfully confirmed our second hypothesis, i.e., intolerance of uncertainty is positively correlated with social anxiety level. This result further strengthens the current construct in the literature that intolerance of uncertainty is one of the sub-traits of social anxiety or social phobia disorder. As expected, the proposed scale was also negatively correlated with the third validating scale, SACQ, which means $\mathrm{PB}$ is not necessarily related to social anxiety. This result contradicts findings in the literature on PB, which requires social interaction (Carlo et al., 2004). While PB is argued to be positively correlated with sub-traits that are negatively correlated with social anxiety, the results of this study suggest otherwise. This contradiction with previous studies is related to the number of items posted in the pro-social questionnaire. However, the new SAS can be considered valid for measuring social anxiety since it correlated with the three other validating scales.

The reliability scores of the proposed scale can be considered satisfactory as it has excellent internal consistency, which means each item was perceived by the participants in the way we intended. In addition, the item discrimination indices shown in Table 2 suggest that most of the items pass the discriminant index cut-off score. Item \#6 ("I feel tense if I am alone with just one other person") was an exception to these findings, thereby suggesting that the item may not be specific in determining or identifying social anxiety traits or its sub-traits. We believe that the wording of this item misled participants to consider an awkward social situation that most people 
would consider normal instead of one that triggers social anxiety. On the other hand, items \#4 (I feel stressed when I have to talk in front of a group) and \#9 (I often worry how to greet someone) showed the highest discriminant indices, likely because of how specific the wording of the statements was. Item \#4, for example, specifically asked if the participants "feel stressed" when engaging in public speaking. This item addresses how socially anxious people feel about talking to a group and becoming the center of attention, similar to a corresponding item in the DSM V (American Psychiatric Association, 2013; Antony \& Swinson, 2000; Mattick \& Clarke, 1998).

The proposed scale was constructed by comprehensive analysis of several existing scale available in the literature. In doing so, the scale may be considered to present multiple perspectives on social anxiety measurement. Our scale is also simpler compared with previous measures as it only has 10 items but features good reliability and satisfactory validity. This study presents some limitations. For example, the questionnaires were distributed along with other questionnaires that were not relevant to the present study. Some of the participants may have encountered exhaustion as they had to fill out numerous questionnaires. We also acknowledge that a non-clinical sample may be inapplicable to most settings. This is due to the nature of a broader approach to social anxiety, especially in our setting (for academic setting and college students). Another consideration to note is that this study may not completely represent social anxiety traits and sub-traits. Other aspects related to social phobia or other confounding effects that may influence an individual's social behavior and perception may exist, and we cannot account for these items. As we limited the validating measures to only three other scales, the construct may be slightly different than the original one.

In future work, the developed scale can be improved by changing or revising some of the items with low item discrimination indices. Should a newer and revised measurement of social anxiety be published, we suggest setting a more conservative score for its item discrimination indices. By specifying a more conservative approach to the scale, better and more-accurate items can provide better measurements of social anxiety. We also suggest the use of a validated and reliable PB construct and measurement scale to scrutinize the related relationships. We also suggest that future research on discriminating social anxiety in the clinical setting and clinical population be conducted. We believe that the clinical setting can provide a better understanding of social anxiety and better interventions than the non-clinical setting. Finally, further contrast studies may provide a better understanding of this measure and determining social anxiety. We recommend replicating this study with contrast conditions to investigate social phobia in various setting or environments.

\section{References}

American Psychiatric Association. (2013). Diagnostic and Statistical Manual of Mental Disorder (5th ed.). Arlington, VA: American Psychiatry Publishing.

Antony, M. M., \& Swinson, R. P. (2000). Phobic disorders and panic in adults: A guide to assessment and treatment. Washington, DC: American Psychological Association.

Ary, D., Jacob, L. C., Irvine, C. K. S., \& Walker, D. (2013). Introduction to research in education $\left(9^{\text {th }}\right.$ ed). Belmont, CA: Wadsworth, Cengage Learning.

Baker, R. W., \& Siryk, B. (1989). Student Adaptation to College Questionnaire. Los Angeles, CA: Western Psychological Services.

Carleton, R. N., Norton, P. J., \& Asmundson, G. J. (2007). Fearing the unknown: A short version of the Intolerance of Uncertainty Scale. Journal of Anxiety Disorders, 21(1), 105-117. doi:10.1016/j.janxdis.2006.03.014 
Carlo, G., Okun, M. A., Knight, G. P., \& Guzman, M. R. (2005). The interplay of traits and motives on volunteering: Agreeableness, extraversion and prosocial value motivation. Personality and Individual Differences, 38(6), 1293-1305. doi:10.1016/j.paid.2004.08.012

Credé, M., \& Niehorster, S. (2012). Adjustment to college as measured by the Student Adaptation to College Questionnaire: A quantitative review of its structure and relationships with correlates and consequences. Educational Psychology Review, 24(1), 133-165. doi:10.1007/s10648-011-9184-5

Cronbach, L. (1951). Coefficient alpha and the internal consistency of tests. Psychometrika, 16, 297-334.

Culotta, C. M., \& Goldstein, S. E. (2008). Adolescents' aggressive and prosocial behavior: Associations with jealousy and social anxiety. The Journal of Genetic Psychology, 169(1), 21-33.

Eysenck, H. J. (1958). A short questionnaire for the measurement of two dimensions of personality. Journal of Applied Psychology, 42(1), 14-17. doi:10.1037/h0041738

Graziano, W. G., \& Eisenberg, N. H. (1997). Agreeableness: A dimension of personality. In R. Hogan, J. A. Johnson, \& S. R. Briggs (Eds.), Handbook of personality psychology (pp. 795-824). San Diego, CA. Academic.

Kaplan, S. C., Levinson, C. A., Rodebaugh, T. L., Menatti, A., \& Weeks, J. W. (2015). Social anxiety and the Big Five Personality Traits: The interactiverelationship of trust and openness. Cognitive Behaviour Therapy, 44(3), $212-222$. doi:10.1080/16506073.2015.1008032

Kumaraswamy, N. (2013). Academic stress, anxiety and depression among college Students- A brief review. International Review of Social Sciences and Humanities, [online] 5(1), pp.135-143. Available at: http://www.irssh.com/.

Mattick, R. P., \& Clarke, J. (1998). Development and validation of measures of social phobia scrutiny fear and social interaction anxiety. S.T. Behaviour Research and Therapy,36(4), 455-470. doi:10.1016/s0005-7967(97)10031-6

Peters, L. (2000). Discriminant validity of the Social Phobia and Anxiety Inventory (SPAI), the Social Phobia Scale (SPS) and the Social Interaction Anxiety Scale (SIAS). Behaviour Research and Therapy, 38(9), 943-950. doi:10.1016/s00057967(99)00131-x

Santos, L., Loureiro, S., Crippa, J., \& Osório, F. (2015). Can the Liebowitz Social Anxiety Scale - Self-Report Version be used to differentiate clinical and non-clinical SAD groups among Brazilians? PLOS ONE, 10(3), p. e0121437.

Turner, S. M.; Beidel, D. C.; \& Dancu, C. V. (1996). Social Phobia and Anxiety Inventory: Manual. Toronto, Ontario: MultiHealth System. 